\section{Cardiotoxicity of tyrosine kinase inhibitors in chronic myelogenous leukemia therapy}

\author{
Zhenshu Xu, ${ }^{1,2}$ Shundong Cang,' \\ Ting Yang, ${ }^{2}$ Delong Liu' \\ 'Division of Hematology and Oncology, \\ New York Medical College, \\ Valhalla, NY, USA \\ 2Department of Hematology, Institute of \\ Hematology, Union Hospital, Fujian \\ Medical University, Fuzhou, China
}

\begin{abstract}
Emerging evidence suggests that the three tyrosine kinase inhibitors currently approved for the treatment of patients with chronic myelogenous leukemia (CML) - imatinib, dasatinib, and nilotinib - have potential cardiotoxic effects. The mechanisms behind these events, and the relations between them, are largely unclear. For example, relative to dasatinib and nilotinib, severe congestive heart failure and left ventricular dysfunction are rare but prominent with imatinib treatment, particularly in patients receiving higher doses (>600 mg/day). In comparison with imatinib, prolongation of the QT interval is relatively common in patients treated with either dasatinib or nilotinib. In contrast to nilotinib, pericardial effusions are observed with both imatinib and dasatinib. It is suggested that these data, an evaluation of cardiac status, use of concomitant medications, and potential risk factors should be considered in the management of CML.
\end{abstract}

\section{Introduction}

BCR-ABL tyrosine kinase is a key molecule responsible for the pathophysiology of chronic myelogenous leukemia (CML). Tyrosine kinase inhibitors (TKIs) directed against BCR$\mathrm{ABL}$ are currently the cornerstone of treatment for patients with CML. Imatinib (Gleevec ${ }^{\circledast}$; Novartis Pharmaceuticals Corporation, New Jersey, USA) was the first TKI approved for CML. Imatinib has marked response and survival benefits over interferon- $\alpha$ plus low-dose cytarabine and is presently the only TKI licensed for first-line treatment. ${ }^{1,2}$ However, despite the remarkable success of imatinib, many patients discontinue treatment because of either resistance or intolerance to this drug.

In the pivotal phase III IRIS (International Randomized Study of Interferon and STI571) trial, primary resistance was observed in $24 \%$ of patients, and secondary resistance presented as relapse in $17 \%$ of patients and disease progression in 7\% after 4.5 years. ${ }^{2}$ After six years of follow-up, $34 \%$ of patients had discontinued imatinib treatment, mostly (14\%) because of an unsatisfactory therapeutic effect (defined as lack of efficacy/progression), but a number of patients (5\%) also stopped receiving the drug as a result of adverse events (AEs) or abnormal laboratory values. ${ }^{3}$ Further treatment options therefore continue to be developed.

Second-line choices for treatment include increasing the dose of imatinib, or changing therapy to dasatinib [Sprycel ${ }^{\oplus}$; Bristol-Myers Squibb Co. (BMS), New York, USA] or nilotinib (Tasigna ${ }^{\oplus}$; Novartis Pharmaceuticals Corp., New Jersey, USA). Newer agents for imatinib failures are still under clinical development. ${ }^{4}$ Dasatinib potently inhibits BCR-ABL. ${ }^{5}$ Compared with imatinib, dasatinib has 325fold greater activity against native BCR-ABL. ${ }^{6}$ Furthermore, dasatinib inhibits all imatinibresistant mutations of this molecule (key mediators of imatinib resistance) except the T315l mutant, which is resistant to all currently available TKIs. $^{6-8}$ It is, however, relatively insensitive to the F317L mutation. ${ }^{9}$

Dasatinib was originally approved at the dosage of $70 \mathrm{mg}$ twice daily, following data from the START (the SRC/ABL Tyrosine Kinase Inhibition Activity: Research Trials of Dasatinib) program of clinical studies. ${ }^{10-12}$ In November 2007, the label for dasatinib was changed to include updated dosing information, safety information from more than 2,100 patients, and data from a randomized comparison with high-dose imatinib. ${ }^{13}$ The recommended starting dose for patients with chronic phase (CP) CML is now $100 \mathrm{mg}$ once daily. The starting dose for advanced disease remains $70 \mathrm{mg}$ twice daily.

Nilotinib (an analog of imatinib) is also active against all imatinib-resistant BCR-ABL mutations except T315l. ${ }^{14}$ However, nilotinib is relatively inactive against common mutations in the ATP-binding P-loop domain, ${ }^{4}$ and those at the F359 residue in the catalytic domain, of BCR-ABL. ${ }^{6}$ Patients harboring such mutations at baseline do not respond to nilotinib and progress quickly during treatment. Furthermore, patients who do not have these mutations at baseline may eventually develop them when resistance to imatinib occurs..$^{15}$ Nilotinib has recently been approved by the FDA for treating imatinib-resistant and -intolerant patients with $\mathrm{CP}$ or accelerated phase CML, but not those suffering from blast crisis.

TKIs share a number of common adverse effects (AE), including neutropenia and thrombocytopenia. Cardiotoxicities of the TKIs have become a clinical concern. This review provides an overview of common AEs and cardiotoxicities associated with these TKIs.
Correspondence: Delong Liu,

Associate Professor of Medicine,

Division of Hematology and Oncology,

New York Medical College,

Valhalla, NY 10595, USA

E-mail: delong_liu@nymc.edu

Key words: tyrosine kinase inhibitors, imatinib, dasatinib, nilotinib, cardiotoxicity.

Acknowledgments: ZX and SC are CAHON Research Scholars (Chinese American Hematologists Oncologist Network, www.cahon.org).

$\mathrm{SC}$ is a recipient of a fellowship grant from the International Scholar Exchange Foundation. This work was partly supported by New York Medical College Blood Diseases Fund. Writing and editorial support were provided by Zoila Mora and Josh Collis, and funded by Bristol-Myers Squibb.

Received for publication: 13 January 2009

Revision received: 6 March 2009

Accepted for publication: 11 March 2009

This work is licensed under a Creative Commons Attribution 3.0 License (by-nc 3.0).

(OCopyright Z. Xu et al., 2009

Licensee PAGEPress, Italy

Hematology Reviews 2009; 1:e4

doi:10.4081/hr.2009.e4

\section{Common adverse events associated with tyrosine kinase inhibitors}

A number of AEs are associated with TKIs. The most common ones are hematologic. In the IRIS study, anemia, neutropenia, and thrombocytopenia (all grades) were each reported by $45-61 \%$ of patients receiving imatinib. ${ }^{1}$ Grade 3-4 anemia, neutropenia, and thrombocytopenia were observed in $3 \%, 14 \%$, and $8 \%$ of patients, respectively. The updated dasatinib label includes data from the START-R trial, a comparison of dasatinib $70 \mathrm{mg}$ twice daily with high-dose imatinib $(800 \mathrm{mg} /$ day $)$ in patients with CP CML resistant to standarddose imatinib. ${ }^{13,16}$ In this study, the incidences of grade 3-4 thrombocytopenia and neutropenia for high-dose imatinib were $14 \%$ and $39 \%$, respectively. The START-R study also showed that dasatinib has significant response and progression-free survival benefits compared with high-dose imatinib, and that significantly more patients discontinue high-dose imatinib than dasatinib. ${ }^{16}$

The $100 \mathrm{mg}$ once daily dose for dasatinib in patients with CP CML was approved as a result of data from a phase III dose modification study. ${ }^{17}$ Shah and colleagues compared dasatinib $100 \mathrm{mg}$ once daily, $50 \mathrm{mg}$ twice daily, 140 $\mathrm{mg}$ once daily, and $70 \mathrm{mg}$ twice daily doses in CP CML. In this study, response rates were 
similar between doses, and overall efficacy was equivalent between the $70 \mathrm{mg}$ twice daily and $100 \mathrm{mg}$ once daily regimens. In the $100 \mathrm{mg}$ once daily dose there was significantly less grade 3 -4 thrombocytopenia $(22 \% ; p=0.004)$ compared with the $70 \mathrm{mg}$ twice daily dose (37\%). Grade 3-4 anemia, leukopenia, and neutropenia were observed in $10 \%, 16 \%$ and $33 \%$ of patients, respectively. The $100 \mathrm{mg}$ once daily dose was also associated with the lowest frequencies of treatment discontinuation (overall: 16\% vs. 23\%; toxicity alone: $4 \%$ vs. $11 \%$, respectively).

For nilotinib, the incidence rates of grade 3 4 thrombocytopenia (28\%), neutropenia (28\%), and anemia (8\%) at the recommended dose $(800 \mathrm{mg} /$ day) in patients with CP CML appear to be similar to those for dasatinib 100 mg once daily. ${ }^{17,18}$

The incidences of non-hematologic AEs are much lower than those for hematologic events for all TKIs, and are broadly similar between TKIs at their current recommended doses. Cutaneous toxicity is more common for TKIs against receptor tyrosine kinases. ${ }^{19}$

\section{Cardiotoxicities observed in chronic myeloid leukemia}

Current evidence suggests that TKIs have potential cardiotoxic effects. Cardiac AEs reported include palpitations, arrhythmia, QT prolongation, pericardial effusions, myocardial ischemia, myocardial infarction, and congestive heart failure (CHF). All of the clinically available BCR-ABL inhibitors report the potential for cardiotoxicity in their respective package inserts (Table 1). ${ }^{13,18,20}$

\section{Imatinib}

Although rare, severe CHF and left ventricular dysfunction have been reported during imatinib treatment, especially in patients with risk factors or comorbidities. In the IRIS trial, this was observed in $0.7 \%$ of patients. ${ }^{1,20}$ Cardiotoxicity associated with high-dose imatinib was not reported during the START-R study. ${ }^{16}$ In smaller studies of high-dose imatinib, existing CHF was exacerbated in one study ( $6 \%$ of patients), and was related to mortality in another ( $4 \%$ of patients). ${ }^{21,22}$

In a large review of patients enrolled in imatinib clinical studies $(n=1,276), 1.8 \%$ of patients had symptoms suggestive of systolic heart failure. Most had risk factors for cardiac failure. In total, $0.6 \%$ of patients had cardiac events attributed to imatinib treatment. The authors concluded that CHF is rare during imatinib treatment. ${ }^{23} \mathrm{~A}$ similar incidence of CHF (1\%) was reported in a nine-year retrospective review at a single institution. ${ }^{24}$ Reports of smaller studies also confirm that cardiac failure is a rare feature of imatinib therapy. ${ }^{25,26}$

The mechanism underlying imatinibinduced cardiac failure is currently unclear. In an in vitro study, physiological concentrations of imatinib significantly and adversely affected mitochondrial membrane potential, apoptosis, cell viability, and cellular ultrastructure. ${ }^{27}$ This cardiotoxic effect may be linked to inhibition of BCR-ABL. Imatinib was reported to cause stress-induced and dose-dependent mitochondrial changes in murine ventricular myocytes, which was reduced by re-engineering the imatinib molecule such that BCR-ABL inhibition was hampered. ${ }^{28,29}$ Nonetheless, the re-engineered molecule may have had altered activities besides reduced BCR-ABL inhibition.

A second cardiac AE associated with imatinib therapy is fluid retention manifesting as pericardial effusion. Grade 3-4 fluid retention reactions, which included pericardial effusions, were reported in $2 \%$ of patients in the IRIS study and in $6 \%$ of all other CML clinical studies. ${ }^{20}$ Frank pericarditis has been observed in $<0.1 \%$ of patients receiving imatinib (all indications). ${ }^{20}$ Other cardiac AEs include tachycardia, hypertension, hypotension, flushing, and peripheral coldness, were each reported in $0.1-1.0 \%$ of patients. ${ }^{20}$

Precautions and general guidelines for dose adjustment for cardiac AEs associated with imatinib treatment are included in the prescribing information and have been summarized in Table 2. CML patients with existing cardiac disease or cardiac risk factors should be monitored and treated accordingly. ${ }^{20,23}$ Patients should also be weighed regularly and monitored for signs and symptoms of fluid retention. Unexpected weight gain should be investigated carefully, and treated appropriately. ${ }^{20,30}$ Significant fluid retention (local or general) can usually be managed by interrupting imatinib treatment and using diuretics or other supportive care.$^{20}$ In severe cases of fluid retention, imatinib should be withheld until this is resolved.

\section{Nilotinib}

Nilotinib can cause QT prolongation and sudden death and carries a black box warning for these side effects. ${ }^{18}$ Nilotinib prolongs the QT interval in a concentration-dependent manner, and the common AEs occurred in 1$10 \%$ of all patients in clinical trials. ${ }^{18}$ In a study in healthy volunteers, nilotinib was associated with a maximum mean QT interval increase of $18 \mathrm{~ms}$ (adjusted for placebo). ${ }^{18}$ In imatinibresistant patients with CML, nilotinib caused a maximum mean QT change of $10 \mathrm{~ms}$ from baseline; QT increase $>60 \mathrm{~ms}$ and QT $>500 \mathrm{~ms}$ associated with nilotinib were reported in $2.1 \%$ and $<1 \%$ of patients, respectively. Sudden deaths were reported in $0.6 \%$ of patients in a clinical study, and at a similar frequency in an expanded access study. The early occurrences of some of these deaths relative to the start of nilotinib treatment suggest that ventricular repolarization may have contributed to their occurrence. $^{18}$

Another common cardiac AE associated with nilotinib therapy is myocardial ischemia. In the pivotal phase II study, it was reported in $7 \%$ (21/321) of patients with CP CML receiving nilotinib as second-line treatment. ${ }^{31}$ Uncommon cardiac events $(0.1-1 \%$ of all patients in clinical trials) include cardiac failure, angina pectoris, atrial fibrillation, pericardial effusion, coronary artery disease, cardiomegaly, cardiac murmur, and bradycardia. Rare events (of uncertain frequency) include myocardial infarction, ventricular dysfunction, pericarditis, cardiac flutter, and extrasystoles. ${ }^{18}$

The mechanisms underlying these AEs are still unclear. In a manner similar to imatinib, nilotinib was found to decrease the cellular viability of rat cardiomyocytes cultured in vitro, although the integrity of the mitochondrial membrane potential was unaffected. ${ }^{27}$ However, nilotinib has also been found to inhibit human ether-a-go-go related gene ( $h E R G)$ potassium currents with an $\mathrm{IC}_{50}$ of $0.66 \mu \mathrm{M}$. This concentration is approximately one-tenth the expected Cmax for this compound, well within therapeutic levels. This mechanism is likely to underlie nilotinibinduced QT prolongation. Inhibition of hERG channels is established as a cause of QT prolongation for a number of compounds, and is a significant barrier in the development of new drugs. ${ }^{32}$ Indeed, the phase II development of the aurora kinase inhibitor MK-0457 (VX-680) was recently suspended, pending a full analysis of all efficacy and safety data. The decision was based on preliminary safety data, in which QT prolongation was observed in one patient. ${ }^{33}$

The potential for QT prolongation and sudden death associated with nilotinib, although rare, necessitates vigilant monitoring. In particular, ECGs should be performed at baseline, seven days after initiation of treatment, periodically throughout therapy, and following dose adjustments. Electrolyte levels should be monitored periodically throughout therapy. Nilotinib is contraindicated for patients with hypokalemia, hypomagenesmia or long QT syndrome. ${ }^{18}$ The nilotinib prescribing information recommends dose adjustments for QT prolongation, presented in Table 2.

\section{Dasatinib}

The events of dasatinib-induced QT prolongation are rare although a warning for such a possible event is given. In single-arm studies of dasatinib, nine patients (1\%) had QT prolongation reported as an $\mathrm{AE} .{ }^{13}$ The mean $\mathrm{QT}$ 
Table 1. Reported incidence of cardiotoxicity during tyrosine kinase inhibitors treatment.

\begin{tabular}{|c|c|c|c|}
\hline Toxicity & $\mathrm{n}$ & Incidence (\%) & Reference \\
\hline \multicolumn{4}{|l|}{ Imatinib } \\
\hline Pericardial effusions & NR & 6 & Novartis $^{20}$ \\
\hline Systolic heart failure & 1276 & 1.8 & Atallah $e t a l^{3}$ \\
\hline Congestive heart failure & 553 & 0.7 & Novartis $^{20}$ \\
\hline Left ventricular dysfunction & 553 & 0.7 & Novartis $^{20}$ \\
\hline Cardiac failure & NR & $0.1-1.0$ & Novartis ${ }^{20}$ \\
\hline Flushing & NR & $0.1-1.0$ & Novartis ${ }^{20}$ \\
\hline Hypertension & NR & $0.1-1.0$ & Novartis ${ }^{20}$ \\
\hline Hypotension & NR & $0.1-1.0$ & Novartis ${ }^{20}$ \\
\hline Peripheral coldness & NR & $0.1-1.0$ & Novartis $^{20}$ \\
\hline Tachycardia & NR & $0.1-1.0$ & Novartis $^{20}$ \\
\hline Pericarditis & NR & $<0.1$ & Novartis ${ }^{20}$ \\
\hline \multicolumn{4}{|l|}{ Dasatinib } \\
\hline Severe pericardial effusions & NR & 1 & $\mathrm{BMS}^{13}$ \\
\hline Congestive heart failure & 911 & 4 & Brave et al..$^{34}$ \\
\hline Arrhythmia & NR & $1-<10$ & $\mathrm{BMS}^{13}$ \\
\hline Palpitations & NR & $1-<10$ & $\mathrm{BMS}^{13}$ \\
\hline QT prolongation > $500 \mathrm{~ms}$ & -300 & $<1$ & $\mathrm{BMS}^{13}$ \\
\hline Angina pectoris & NR & $0.1-<1$ & $\mathrm{BMS}^{13}$ \\
\hline Cardiomegaly & NR & $0.1-<1$ & $\mathrm{BMS}^{13}$ \\
\hline Myocardial infarction & NR & $0.1-<1$ & $\mathrm{BMS}^{13}$ \\
\hline Pericarditis & NR & $0.1-<1$ & $\mathrm{BMS}^{13}$ \\
\hline Ventricular arrhythmia & NR & $0.1-<1$ & $\mathrm{BMS}^{13}$ \\
\hline Acute coronary syndrome & NR & $<0.1$ & $\mathrm{BMS}^{13}$ \\
\hline Myocarditis & NR & $<0.1$ & $\mathrm{BMS}^{13}$ \\
\hline \multicolumn{4}{|l|}{ Nilotinib } \\
\hline Myocardial ischemia & 321 & 7 & Kantarjian et $a l^{31}$ \\
\hline Increase in QTcF > $60 \mathrm{~ms}$ from BL & 232 & 2.1 & Novartis ${ }^{18}$ \\
\hline Palpitations & NR & $1-10$ & Novartis ${ }^{18}$ \\
\hline Angina pectoris & NR & $0.1-1$ & Novartis $^{18}$ \\
\hline Atrial fibrillation & NR & $0.1-1$ & Novartis $^{18}$ \\
\hline Bradycardia & NR & $0.1-1$ & Novartis $^{18}$ \\
\hline Cardiac failure & NR & $0.1-1$ & Novartis $^{18}$ \\
\hline Cardiac murmur & NR & $0.1-1$ & Novartis ${ }^{18}$ \\
\hline Cardiomegaly & NR & $0.1-1$ & Novartis ${ }^{18}$ \\
\hline Coronary artery disease & NR & $0.1-1$ & Novartis ${ }^{18}$ \\
\hline Pericardial effusion & NR & $0.1-1$ & Novartis $^{18}$ \\
\hline Death $^{\mathrm{a}}$ & 867 & 0.6 & Novartis ${ }^{18}$ \\
\hline
\end{tabular}

$B L$, baseline; NR, not reported, representing all patients within each agent's clinical trials. ${ }^{P}$ Possibly resulting from ventricular repolarization.

interval increased by 3-6 ms (Fridericia's method); this increase was not clinically relevant. $^{34}$ In total, $<1 \%$ of patients had a QT increase to $>500 \mathrm{~ms}$. In contrast with nilotinib, the $\mathrm{IC}_{50}$ for dasatinib for the inhibition of hERG currents $(14.3 \mu \mathrm{M})$ is 100 times the expected Cmax for this drug. ${ }^{27}$ This may explain why QT prolongation is more clinically prominent for nilotinib than it is for dasatinib.

Common cardiac AEs (observed in $1-<10 \%$ of all patients in clinical trials) include arrhythmia and palpitations. Severe pericardial effusions have been reported in $1 \%$ of all patients in all clinical studies, and the prescribing information for dasatinib includes a warning for this toxicity. ${ }^{13}$ Severe CHF has also been reported in $1 \%$ of all patients. ${ }^{13}$ In singlearm studies, CHF or ventricular dysfunction occurred in $4 \%(20 / 911)$ of patients. ${ }^{34}$ However, in the dose optimization study, dasatinib $100 \mathrm{mg}$ once daily was not associated with any incidence of severe CHF or pericar- dial effusion in any patient; both AEs were reported in patients receiving the $70 \mathrm{mg}$ twice daily dose (all grades, $4 \%$; grade $3-4,3 \%){ }^{17}$

Both pericardial effusions and cardiac failure associated with dasatinib therapy may be caused by similar mechanisms to those associated with imatinib treatment, although, in contrast with imatinib, dasatinib has not been found to significantly affect mitochondrial membrane potential, apoptosis, cell viability, or cellular ultrastructure at physiological con- 
Table 2. Precautions and dose modifications on the emergence of cardiac events during tyrosine kinase inhibitors treatment.

\begin{tabular}{|c|c|c|}
\hline Agent & Dose modification & Precautions \\
\hline Imatinib $^{20}$ & $\begin{array}{l}\text { On the emergence of a severe event, withhold until } \\
\text { the event has resolved. Resume at an appropriate dose, } \\
\text { depending on initial severity of event. }\end{array}$ & $\begin{array}{l}\text { Carefully monitor any patient with or at risk of cardiac failure. } \\
\text { All patients with signs or symptoms } \\
\text { of cardiac failure should be evaluated and treated. }\end{array}$ \\
\hline Dasatinib $^{13}$ & $\begin{array}{l}\text { On the emergence of a severe event, withhold until the } \\
\text { event has resolved or improved. Resume at an } \\
\text { appropriate dose, depending on initial severity of event. }\end{array}$ & $\begin{array}{l}\text { Administer with caution to patients with or at risk of QTc prolongation: } \\
\text { those with hypokalemia, hypomagnesemia, or congenital long QT } \\
\text { syndrome; or taking medicines known to prolong QT, including } \\
\text { anti-arrhythmic drugs, and cumulative high-dose anthracycline therapy } \\
\text { Correct hypokalemia or hypomagnesemia prior to administration. }\end{array}$ \\
\hline Nilotinib $^{18}$ & $\begin{array}{l}\text { QTc }>480 \text { ms: 1) withhold therapy, correct serum } \\
\text { potassium and magnesium levels if below normal, } \\
\text { and review concomitant medication; } 2 \text { ) resume at prior dose in } \\
<2 \text { weeks if QTc returns to }<450 \mathrm{~ms} \text { and }<20 \text { ms of baseline; } \\
\text { 3) reduce dose to } 400 \mathrm{mg} / \text { day if QTc } 450-480 \mathrm{~ms} \text { after } 2 \text { weeks; } \\
\text { 4) discontinue if QTc returns to }>480 \mathrm{~ms} \text { after dose reduction; } \\
\text { 5) repeat ECG assessment approx. } 7 \text { days after any dose adjustment. }\end{array}$ & $\begin{array}{l}\text { Do not administer to patients with long QT syndrome. } \\
\text { Do not administer drugs known to prolong QT, } \\
\text { and strong CYP3A4 inhibitors. } \\
\text { Correct hypokalemia or hypomagnesemia prior to administration, } \\
\text { and periodically monitor serum electrolyte levels during therapy. } \\
\text { Perform ECGs at baseline, } 7 \text { days after treatment starts, } \\
\text { periodically as indicated clinically, and after any dose adjustment. }\end{array}$ \\
\hline
\end{tabular}

centrations. $^{27}$

Uncommon cardiac AEs associated with dasatinib include angina pectoris, cardiomegaly, pericarditis, ventricular arrhythmia, and myocardial infarction (reported in $0.1-<1 \%$ of patients). Rare events (reported in $<0.1 \%$ of patients) include myocarditis and acute coronary syndrome. ${ }^{13}$

Dasatinib should be administered with caution to any patient at risk of cardiac problems (especially prolongation of the QT interval). Patients with pre-existing congenital long QT syndrome or those receiving anti-arrhythmic medicine should be monitored closely. ${ }^{13}$ Patients with hypokalemia or hypomagnesemia should have these conditions corrected prior to receiving dasatinib. As several antineoplastic agents, including the anthracyclines, are associated with cardiotoxicity, the treatment history of a patient should also be taken into consideration before commencing any therapy. Fluid retention events can typically be managed by supportive measures. Dose interruption is also indicated (Table 2).$^{13}$

\section{Conclusions}

Although rare, severe CHF and left ventricular dysfunction have occurred with imatinib treatment, especially in patients receiving higher doses. Cardiac failure is also an uncommon feature of nilotinib and dasatinib therapy. However, CHF has not yet been reported in patients with CP CML receiving dasatinib with a starting dose of $100 \mathrm{mg}$ once daily. Compared with imatinib, palpitations and prolongation of the QT interval are relatively common with both dasatinib and nilotinib. Nilotinib carries a black box warning for such AE and sudden death has been observed. These AEs may be related to BCR-ABL inhibition and therefore be genuine class effects. Further research is needed to clarify the mechanisms underlying these effects.

\section{References}

1. O'Brien SG, Guilhot F, Larson RA, et al. Imatinib compared with interferon and low-dose cytarabine for newly diagnosed chronic-phase chronic myeloid leukemia. N Engl J Med 2003;348:994-1004.

2. Druker BJ, Guilhot F, O'Brien SG, et al. Five-year follow-up of patients receiving imatinib for chronic myeloid leukemia. $\mathrm{N}$ Engl J Med 2006;355: 2408-17.

3. Hochhaus A, Druker BJ, Larson RA, 0'Brien SG, Gathmann I, Guilhot F. IRIS 6year follow-up: sustained survival and declining annual rate of transformation in patients with newly diagnosed chronic myeloid leukemia in chronic phase (CMLCP) treated with imatinib. Blood 2007;110: Abstract 25.

4. Cang S, Liu D. P-Loop mutations and novel therapeutic approaches for imatinib failures in chronic myeloid leukemia. J Hematol Oncol 2008;1:15.

5. Lombardo LJ, Lee FY, Chen P, et al. Discovery of N-(2-chloro-6-methylphenyl)-2-(6-(4-(2-hydroxyethyl)- piperazin-1-yl)-2-methylpyrimidin-4- ylamino) thiazole-5-carboxamide (BMS-354825), a dual Src/Abl kinase inhibitor with potent antitumor activity in preclinical assays. $\mathrm{J}$ Med Chem 2004;47:6658-61.

6. O'Hare T, Walters DK, Stoffregen EP, et al. In vitro activity of Bcr-Abl inhibitors AMN107 and BMS-354825 against clinically relevant imatinib-resistant Abl kinase domain mutants. Cancer Res 2005;65: 4500-5.

7. Shah NP, Tran C, Lee FY, Chen P, Norris D, Sawyers CL. Overriding imatinib resistance with a novel ABL kinase inhibitor. Science 2004;305:399-401.

8. Tokarski JS, Newitt JA, Chang CY, et al. The structure of dasatinib (BMS-354825) bound to activated ABL kinase domain elucidates its inhibitory activity against imatinib-resistant ABL mutants. Cancer Res 2006;66:5790-7.

9. Soverini S, Colarossi S, Gnani A, et al. Resistance to dasatinib in Philadelphiapositive leukemia patients and the presence or the selection of mutations at residues 315 and 317 in the BCR-ABL kinase domain. Haematologica 2007;92: 401-4.

10. Cortes J, Rousselot P, Kim DW, et al. Dasatinib induces complete hematologic and cytogenetic responses in patients with imatinib-resistant or -intolerant chronic myeloid leukemia in blast crisis. Blood 2007;109:3207-13.

11. Guilhot F, Apperley J, Kim DW, et al. Dasatinib induces significant hematologic and cytogenetic responses in patients with imatinib-resistant or -intolerant chronic myeloid leukemia in accelerated phase. Blood 2007;109: 4143-50.

12. Hochhaus A, Kantarjian HM, Baccarani M, et al. Dasatinib induces notable hematologic and cytogenetic responses in chronic phase chronic myeloid leukemia after failure of imatinib therapy. Blood 2007;109: 2303-9.

13. Bristol-Myers Squibb Company. Dasatinib prescribing information. Bristol-Myers Squibb Company, 2007. Available at: http://www.bms.com/products/data/

14. Weisberg E, Manley PW, Breitenstein W, et 
al. Characterization of AMN107, a selective inhibitor of native and mutant Bcr-Abl. Cancer Cell 2005;7:129-41.

15. Branford S, Shou Y, Lawrence R, Rudzki Z, Hughes T. The P-loop mutations Y253H and E255K/V may develop more frequently than T3151 during nilotinib therapy after imatinib failure and are associated with progression in patients with $\mathrm{Ph}$-positive leukemia. Haematologica 2007;92(S1): 337.

16. Kantarjian H, Pasquini R, Hamerschlak N, et al. Dasatinib or high-dose imatinib for chronic-phase chronic myeloid leukemia after failure of first-line imatinib: a randomized phase 2 trial. Blood 2007;109: 5143-50.

17. Shah NP, Kantarjian HM, Kim DW, et al. Intermittent target inhibition with dasatinib $100 \mathrm{mg}$ once daily preserves efficacy and improves tolerability in imatinibresistant and -intolerant chronic-phase chronic myeloid leukemia. J Clin Oncol 2008;26:3204-12.

18. Novartis Pharmaceuticals Corporation: Nilotinib prescribing information. Novartis Pharmaceuticals Corporation, 2007. Available at: http:/www.pharma.us. novartis.com/product/pi/pdf/tasigna.pdf.

19. Huang X, Seiter K, Patel S, Ahmed N, Liu D. Severe toxicity of skin rash, fever and diarrhea associated with imatinib: case report and review of skin toxicities associated with tyrosine kinase inhibitors. Drug Design, Development and Therapy 2008 http://www.dovepress.com/getfile.php?fileI $\mathrm{D}=3682$.

20. Novartis Pharmaceuticals Corporation: Imatinib prescribing information. Novartis Pharmaceuticals Corporation, 2007. Available at: http://www.pharma.us.novar- tis.com/product/pi/pdf/gleevec_tabs.pdf

21. Kantarjian HM, Talpaz M, O'Brien S, et al. Dose escalation of imatinib mesylate can overcome resistance to standard-dose therapy in patients with chronic myelogenous leukemia. Blood 2003;101:473-5.

22. Zonder JA, Pemberton $\mathrm{P}$, Brandt $\mathrm{H}$, Mohamed AN, Schiffer CA. The effect of dose increase of imatinib mesylate in patients with chronic or accelerated phase chronic myelogenous leukemia with inadequate hematologic or cytogenetic response to initial treatment. Clin Cancer Res 2003;9:2092-7.

23. Atallah E, Durand JB, Kantarjian H, Cortes $\mathrm{J}$. Congestive heart failure is a rare event in patients receiving imatinib therapy. Blood 2007;110:1233-7.

24. Breccia M, Cannella L, Frustaci A, Stefanizzi C, Levi A, Alimena G. Cardiac events in imatinib mesylate-treated chronic myeloid leukemia patients: A single institution experience. Leuk Res 2008;32: 835-6.

25. Perik PJ, Rikhof B, de Jong FA, Verweij J, Gietema JA, van der Graaf WT. Results of plasma N-terminal pro B-type natriuretic peptide and cardiac troponin monitoring in GIST patients do not support the existence of imatinib-induced cardiotoxicity. Ann Oncol 2008;19:359-61.

26. Ribeiro AL, Marcolino MS, Bittencourt HN, et al. An evaluation of the cardiotoxicity of imatinib mesylate. Leuk Res 2008;32: 1809-14.

27. Freebern WJ, Fang HS, Slade MD, et al. In vitro cardiotoxicity potential comparative assessments of chronic myelogenous leukemia tyrosine kinase inhibitor therapies: dasatinib, imatinib and nilotinib. Blood 2007;110: Abstract 4582.
28. Kerkelä R, Grazette L, Yacobi R, et al. Cardiotoxicity of the cancer therapeutic agent imatinib mesylate. Nat Med 2006;12: 908-16.

29. Fernandez A, Sanguino A, Peng Z, et al. An anticancer C-Kit kinase inhibitor is reengineered to make it more active and less cardiotoxic. J Clin Invest 2007;117: 4044-54.

30. National Comprehensive Cancer Network: NCCN Clinical Practice Guidelines in Oncology. Chronic myelogenous leukemia V.3.2008. Available at: http://www.nccn.org/ professionals/physician_gls/PDF/cml.pdf.

31. Kantajarian HM, Hochhaus A, Cortes J, et al. Nilotinib is highly active and safe in chronic phase chronic myelogenous leukemia (CML-CP) patients with imatinib-resistance or intolerance. Blood 2007;110: Abstract 735.

32. Pollard CE, Valentin JP, Hammond TG. Strategies to reduce the risk of druginduced QT interval prolongation: a pharmaceutical company perspective. $\mathrm{Br} \mathrm{J}$ Pharmacol 2008;154:1538-43.

33. Vertex Pharmaceuticals Incorporated: Vertex's collaborator Merck suspends patient enrollment in clinical trials of MK0457 (VX-680) pending full analysis of clinical data. Vertex Pharmaceuticals Incorporated, 2007. Available at: http://investors.vrtx.com/releasedetail.cfm ?ReleaseID=276543

34. Brave M, Goodman V, Kaminskas E, et al. Sprycel for chronic myeloid leukemia and Philadelphia chromosome-positive acute lymphoblastic leukemia resistant to or intolerant of imatinib mesylate. Clin Cancer Res 2008;14:352-9. 